\title{
Approximation of mode profile dispersion in the generalized nonlinear Schrödinger equation superior to the constant overlap approach for weakly guiding fibers
}

\author{
Arduin, Andrea; Lægsgaard, Jesper
}

Published in:

Journal of the Optical Society of America B: Optical Physics

Link to article, DOI:

10.1364/JOSAB.432276

Publication date:

2021

Document Version

Peer reviewed version

Link back to DTU Orbit

Citation $(A P A)$ :

Arduin, A., \& Lægsgaard, J. (2021). Approximation of mode profile dispersion in the generalized nonlinear Schrödinger equation superior to the constant overlap approach for weakly guiding fibers. Journal of the Optical Society of America B: Optical Physics, 38(12), 3802-3810. https://doi.org/10.1364/JOSAB.432276

\section{General rights}

Copyright and moral rights for the publications made accessible in the public portal are retained by the authors and/or other copyright owners and it is a condition of accessing publications that users recognise and abide by the legal requirements associated with these rights.

- Users may download and print one copy of any publication from the public portal for the purpose of private study or research.

- You may not further distribute the material or use it for any profit-making activity or commercial gain

- You may freely distribute the URL identifying the publication in the public portal 


\title{
Approximation of mode profile dispersion in the Generalized Nonlinear Schrödinger Equation superior to the constant overlap approach for weakly guiding fibers
}

\author{
Andrea ARduin, ${ }^{1}$ Jesper L/EgsGaARD ${ }^{1,}{ }^{*}$ \\ ${ }^{1}$ DTU Fotonik, Technical University of Denmark, 2800 Kgs. Lyngby, Denmark \\ *jlag@fotonik.dtu.dk
}

\begin{abstract}
An efficient numerical approach to nonlinear propagation in waveguides based on an approximation of the overlap integrals' frequency dependence is proposed. Two test cases are studied, a weakly-guiding step-index fibre and a weakly-guiding parabolic-index fibre, where the approximation is compared to the standard approach of neglecting the frequency dependence and calculating the overlaps at a centre wavelength $\lambda_{0}$, and benchmarked against a GaussianQuadrature approach, whose accuracy can be increased systematically. The combined accuracy and efficiency of the proposed approximation make it superior to the other two approaches in few-mode simulations.
\end{abstract}

(C) 2021 Optical Society of America

\section{Introduction}

Great scientific and technological interest is found in numerical propagation of short pulses in multimoded optical fibres. From a telecommunication point of view, the use of multiple modes might increase the signal transmission capacity of a single core fibre [1]. Moreover, it is well known that high power pulses cause nonlinear frequency conversion through their strong nonlinear interactions. Commercial sources based on this principle are already available and established on the market, and are referred to as Super Continuum sources [2]. A second mechanism that can enable nonlinear frequency conversion is the so-called 'Cherenkov Radiation' or 'Dispersive Wave Generation' (DWG), through which optical power can be resonantly converted to shorter wavelengths [3]. For both these and other methods of nonlinear frequency conversion the use of higher-order modes in multimode fibres holds a great potential for power scaling, due to the possibility of engineering the dispersion properties even in large fibre cores [4,5]. Multimode fibres may also enable new nonlinear phenomena, such as intermodal four-wave mixing [6,7], dispersive-wave generation from spatiotemporal oscillations [8], and beam condensation [9-11]. Interest in numerical modelling of nonlinear propagation is found for example, in [12], which uses machine learning techniques to solve the Nonlinear Schrödinger Equation describing single mode pulse propagation. The standard description of multimode nonlinear propagation uses constant overlap integrals [13], which neglect mode profile dispersion. Both Super Continuum generation and DWG encompass large wavelength ranges, and modelling them using constant overlaps can be source of significant errors. In [14] it was shown that an efficient alternative to the overlap integral method is to evaluate the total field and nonlinear polarization on a reduced transverse grid, allowing to include their frequency dependence with an acceptable complexity penalty. This approach, here denoted Gaussian Quadrature (GQ), works well with smooth mode profiles, and is faster than the overlap method when the number of modes is large. In [15] the two approaches were found to be equivalent with respect to execution time around $M=10$ modes, although the exact $M$ value is problem dependent. In cases where only a few modes of high order are interacting nonlinearly $[5,16]$ the GQ approach is inefficient because the required size of the integration grid depends on the spatial complexity of the modes. It may still be preferable, 
however, due to its superior handling of mode profile dispersion, especially close to modal cutoffs. The purpose of this paper is to propose a modified overlap integral method, based on the approximation of the overlap integrals' frequency dependence through their Effective Areas, labelled in the following as 'EAA' for Effective Area Approximation. The method is tested against the Constant Overlaps (CO) and the GQ for two weakly-guiding few mode fibres: a step-index fibre and a parabolic-index fibre. The proposed approximation would position itself in the few-modes regime, where there is a need to handle mode profile dispersion but the complexity of the GQ is much larger than the constant overlaps. The test scenario is a high-power femtosecond pulse propagating for a short distance, being subject to nonlinear broadening and producing a DWG peak.

\section{Formal Theory}

\subsection{Derivation of the Generalized Nonlinear Schrödinger Equation in the overlap integral formulation}

The Generalized Nonlinear Schrödinger Equation (GNLSE) is discussed and used extensively in the literature to describe high power pulse propagation in optical fibres, as for example in [14], [17] and [18]. The purpose of this section is to briefly introduce the relevant equations, arriving to the overlap integral formulation of the GNLSE.

It is natural to express the fields in a waveguide through an expansion in modes:

$$
\begin{aligned}
& \mathbf{E}(\mathbf{r}, t)=\frac{1}{2 \pi} \sum_{m} \int d \omega G_{m}(z, \omega) \mathbf{e}_{m}\left(\mathbf{r}_{\perp}, \omega\right) \exp \left(i\left(\omega t-\beta_{m}(\omega) z\right)\right) \\
& \mathbf{H}(\mathbf{r}, t)=\frac{1}{2 \pi} \sum_{m} \int d \omega G_{m}(z, \omega) \mathbf{h}_{m}\left(\mathbf{r}_{\perp}, \omega\right) \exp \left(i\left(\omega t-\beta_{m}(\omega) z\right)\right)
\end{aligned}
$$

where $\mathbf{e}_{m}\left(\mathbf{r}_{\perp}\right)$ and $\mathbf{h}_{m}\left(\mathbf{r}_{\perp}\right)$ are the transverse distribution of the modal fields, $G_{m}(z, \omega)$ the complex amplitudes and $\beta_{m}(\omega)$ the propagation constants. Since the fields are real valued in time, it is not necessary to numerically simulate both the positive and negative frequencies, as the transforms possess hermitian symmetry. In Eq. (1) and Eq. (2) this implies that the frequency integral starts at $\omega=0$ instead of $-\infty$ (while the upper boundary is always $+\infty$ ). This is numerically advantageous as it halves the number of frequency grid points required.

It is chosen that the modes are orthogonal and normalized according to

$$
\int d \mathbf{r}_{\perp}\left[\mathbf{e}_{m} \times \mathbf{h}_{n}^{*}+\mathbf{e}_{n}^{*} \times \mathbf{h}_{m}\right]=\delta_{m, n}
$$

where $\delta_{m, n}$ represents the Kronecker delta. The energy in the waveguide can then be found integrating as

$$
E=\int d t P=\sum_{m} \int d t\left|G_{m}(z, t)\right|^{2}
$$

A propagation equation, driven by the nonlinear polarization, can be written as (Eq. (A.15) in [18])

$$
\int d t d \mathbf{r}_{\perp}\left(\mathbf{e}_{m}\left(\mathbf{r}_{\perp}, \omega\right) \exp (i(\omega t-\beta(\omega) z))\right)^{*} \cdot \frac{\partial \mathbf{P}_{N L}}{\partial t}=-\frac{\partial G_{m}(z, \omega)}{\partial z}
$$

where the polarization is still generic. Considering an isotropic material like silica, and neglecting odd (as for example $\left.\chi_{x x x y}^{(3)}\right)$ ) and surface contributions, it can be written as

$$
\mathbf{P}_{N L}(\mathbf{r}, t)=\epsilon_{0} \chi^{(3)}(\mathbf{r}) \mathbf{E}(\mathbf{r}, t) \int d t^{\prime} R\left(\mathbf{r}, t-t^{\prime}\right)\left|\mathbf{E}\left(\mathbf{r}, t^{\prime}\right)\right|^{2}
$$


where $R(\mathbf{r}, t)$ is in general a real valued response function. In silica, the response function comprises of two contributions. One is the electronic contribution, assumed to be instantaneous at optical frequencies, and the other is the slower phononic contribution. These are respectively identified with the Kerr effect and Raman scattering. The response is then written with two terms:

$$
R(t)=\left(1-\tilde{f}_{R}\right) \delta(t)+\tilde{f}_{R} r(t)
$$

where $\delta(t)$ is the Dirac delta, $\tilde{f}_{R}$ is the relative strength of the two effects and $r(t)$ is the Raman only response function, which we model using the parametrization by Hollenbeck and Cantrell [19]. The imaginary part of its Fourier transform peaks at a frequency shift of $\Delta f \approx 13 \mathrm{THz}$ and falls off rapidly for higher frequencies. The strength of the nonlinear response is encoded in $\chi^{(3)}$, so that this function has values comparable to 1. Plugging Eq. (7) in Eq. (6):

$$
\mathbf{P}_{N L}(\mathbf{r}, t)=2 \epsilon_{0} \chi^{(3)}(\mathbf{r})\left(\int d t^{\prime} R\left(t-t^{\prime}\right) \mathbf{E}(t)\left|\mathbf{E}\left(t^{\prime}\right)\right|^{2}\right)+\epsilon_{0} \chi^{(3)}\left(1-f_{R}\right) \mathbf{E}^{*}(t) \mathbf{E}(t) \cdot \mathbf{E}(t)
$$

where it is now understood that only positive frequency components are present. Moreover, two components are neglected: the third harmonic generation term at $\approx 3 \omega_{0}$ and the Raman contribution to the second term. The reasons are respectively the typical strong phase mismatch and the negligible magnitude of the Raman response function at optical frequencies $\approx 2 \omega_{0}$. Restarting from Eq. (5), the polarization term can be written explicitly using Eq. (8). Then, the fields are written using the modal expansion in Eq. (1) and everything is transformed in frequency to reach

$$
\begin{aligned}
\frac{\partial G_{m}(z, \omega)}{\partial z}= & -i \omega \epsilon_{0} \frac{\chi^{(3)}}{(2 \pi)^{2}} \exp \left(i \beta_{m}(\omega) z\right) \sum_{n, p, q} \cdot\{ \\
& \left(2 \iint d \omega_{1} d \omega_{2} \tilde{G}_{n}\left(z, \omega_{1}\right) \tilde{G}_{p}\left(z, \omega_{2}\right) \tilde{G}_{q}^{*}\left(z, \omega_{1}+\omega_{2}-\omega\right) R\left(\omega-\omega_{1}\right) .\right. \\
& \left.\int d \mathbf{r}_{\perp} \mathbf{e}_{m}^{*}\left(\mathbf{r}_{\perp}, \omega\right) \cdot \mathbf{e}_{n}\left(\mathbf{r}_{\perp}, \omega_{1}\right) \mathbf{e}_{p}\left(\mathbf{r}_{\perp}, \omega_{2}\right) \cdot \mathbf{e}_{q}^{*}\left(\mathbf{r}_{\perp}, \omega_{1}+\omega_{2}-\omega\right)\right)+ \\
& \left(\left(1-f_{R}\right) \iint d \omega_{1} d \omega_{2} \tilde{G}_{n}^{*}\left(z, \omega_{1}\right) \tilde{G}_{p}\left(z, \omega_{2}\right) \tilde{G}_{q}\left(z, \omega_{1}+\omega_{2}-\omega\right) .\right. \\
& \left.\left.\int d \mathbf{r}_{\perp} \mathbf{e}_{m}^{*}\left(\mathbf{r}_{\perp}, \omega\right) \cdot \mathbf{e}_{n}^{*}\left(\mathbf{r}_{\perp}, \omega_{1}\right) \mathbf{e}_{p}\left(\mathbf{r}_{\perp}, \omega_{2}\right) \cdot \mathbf{e}_{q}\left(\mathbf{r}_{\perp}, \omega_{1}+\omega_{2}-\omega\right)\right)\right\}
\end{aligned}
$$

where the $\sim$ sign on the $G(z, \omega)$ functions means that they carry the phase factor as in $\tilde{G}_{m}(z, \omega)=$ $G_{m}(z, \omega) \exp \left(-i \beta_{m}(\omega) z\right)$. Assuming the modal transverse integrals are constant in frequency, the Constant Overlaps (CO) formulation becomes

$$
\begin{aligned}
\frac{\partial G_{m}(z, \omega)}{\partial z} & =-i \omega \epsilon_{0} \frac{\chi^{(3)}}{(2 \pi)^{2}} \exp \left(i \beta_{m}(\omega) z\right) \sum_{n, p, q} \cdot\{ \\
& 2 \Gamma_{m, n, p, q}^{(1)} \iint d \omega_{1} d \omega_{2} \tilde{G}_{n}\left(z, \omega_{1}\right) \tilde{G}_{p}\left(z, \omega_{2}\right) \tilde{G}_{q}^{*}\left(z, \omega_{1}+\omega_{2}-\omega\right) R\left(\omega-\omega_{1}\right)+ \\
& \left.\left(1-f_{R}\right) \Gamma_{m, n, p, q}^{(2)} \iint d \omega_{1} d \omega_{2} \tilde{G}_{n}^{*}\left(z, \omega_{1}\right) \tilde{G}_{p}\left(z, \omega_{2}\right) \tilde{G}_{q}\left(z, \omega_{1}+\omega_{2}-\omega\right)\right\}
\end{aligned}
$$

where the two $\Gamma$ factors represent the modal overlaps, and the 1,2 index distinguishes the different conjugations of the four fields. By choosing a basis in which the transverse field components are real, while the $z$-components are purely imaginary, it is easily seen that the difference between the two kinds of $\Gamma$ factor is only due to the $z$-components, which will be small for weak index contrasts, but may be appreciable in waveguides with large index contrast [20]. 


\subsection{Effective Area Approximation of the overlap integrals}

Although the constant overlaps formulation is performing well in some problems, where the power is not distributed over a large spectrum, it is inappropriate in modelling phenomena like supercontinuum generation, which can easily span an octave. The key point lies in the fact that the constant overlaps represent a significant numerical simplification of the GNLSE, whose exact solution is numerically unfeasible, as they allow to solve the double frequency integral through convolutions. Using a Fast Fourier Transform (FFT), a convolution on a frequency grid with $\mathrm{N}$ points has a numerical complexity of $O(N \log N)$, and it can be evaluated for all $\omega$-values at the same time, whereas a generic double-frequency integral evaluated at all $\omega$-values would scale as $O\left(N^{3}\right)$. The objective of the Effective Area Approximation is to extend the constant overlaps, to include a frequency dependence, without losing this property.

[17] proposed and studied an approximation based on the effective area for single mode propagation; this work aims to extend the same principle to a multimode scenario. The proposed approximation of the overlaps is

$$
\begin{gathered}
\int d \mathbf{r}_{\perp} \mathbf{e}_{m}^{*}\left(\mathbf{r}_{\perp}, \omega\right) \cdot \mathbf{e}_{n}\left(\mathbf{r}_{\perp}, \omega_{1}\right) \mathbf{e}_{p}\left(\mathbf{r}_{\perp}, \omega_{2}\right) \cdot \mathbf{e}_{q}^{*}\left(\mathbf{r}_{\perp}, \omega_{1}+\omega_{2}-\omega\right) \approx \\
\frac{f_{m, n, p, q}^{(1)}}{\left(\tilde{A}_{e f f, m}(\omega) \tilde{A}_{e f f, n}\left(\omega_{1}\right) \tilde{A}_{e f f, p}\left(\omega_{2}\right) \tilde{A}_{e f f, q}\left(\omega_{1}+\omega_{2}-\omega\right)\right)^{1 / 4}}
\end{gathered}
$$

with an analogous $f_{m, n, p, q}^{(2)}$ defined for $\Gamma_{m, n, p, q}^{(2)}$. The factors $f_{m, n, p, q}$ are chosen to match exact and approximated overlap integrals when all the frequency arguments are $\omega_{0}$ :

$$
\Gamma_{m, n, p, q}^{(1)}=\frac{f_{m, n, p, q}^{(1)}}{\left(\tilde{A}_{e f f, m}\left(\omega_{0}\right) \tilde{A}_{e f f, n}\left(\omega_{0}\right) \tilde{A}_{e f f, p}\left(\omega_{0}\right) \tilde{A}_{e f f, q}\left(\omega_{0}\right)\right)^{1 / 4}}
$$

where $\omega_{0}$ for example can be the centre frequency of the pulse at the input of the simulation. As shown in the appendix, the photon number is conserved by this approximation. This is also consistent with what was found in [17] for the single mode case. In this work the effective area is defined as

$$
\tilde{A}_{\text {eff }}=\frac{\left[\int \mathbf{d r}_{\perp} \operatorname{Re}\left(\mathbf{e}\left(\mathbf{r}_{\perp}\right) \times \mathbf{h}^{*}\left(\mathbf{r}_{\perp}\right)\right)\right]^{2}}{\epsilon_{0}^{2} c_{0}^{2} \int d r\left|\mathbf{e}\left(\mathbf{r}_{\perp}\right)\right|^{4}}
$$

The definition in Eq. (11) is not dependent on the exact formulation of the effective areas, which could in principle be any kind of function dependent only on one frequency argument and the relative mode index. The advantage in choosing the effective areas is that the overlap integrals of the kind $\Gamma_{m, m, m, m}$ are represented exactly. Different modes tend to drift away from each other during propagation, on account of their different propagation constants, so these terms are among the most important in the spectrum evolution. Since the two proposed test cases are weakly guiding fibres, the distinction between the two conjugations in $\Gamma^{(1)}$ and $\Gamma^{(2)}$ is not applied to the effective areas, and only the one defined in Eq. (12) is used. If necessary, it would be straightforward to define another effective area $A_{e f f}^{\prime}$, with the appropriate field conjungations, to represent $\Gamma^{(2)}$. On the other hand, the two different factors $f_{m, n, p, q}^{(1)}$ and $f_{m, n, p, q}^{(2)}$ are distinguished. This choice is justified by the negligible increase in complexity in doing so, while considering the two different effective areas is expected to roughly double the overhead of the EAA compared to the $\mathrm{CO}$. Running the simulations with the two different effective areas produced in the two test cases a change in the output spectrum $<0.1 \%$. These results were omitted from the experimental section, for the sake of operating a comparison of the three methods in a more practical setting. The assumption of this proposal is that the overlap nature, meant as the way different modes overlap, is not changing strongly with wavelength. For example, the $L P_{1,1}$ does not change the 
number of its lobes or their orientation. Rather, the overlap is decreasing and increasing as the involved modes are respectively expanding and focusing with longer and shorter wavelengths. The effective area is then a way of representing the size of the involved modes. On the other hand, simple products of effective areas cannot capture cancellation effects due to phase profiles of different modes, hence the $f_{m, n, p, q}$ factors are needed. Numerical tests have shown that this approach can be quite accurate in representing the frequency dependence of the overlap integrals of circular symmetric fibres, as will be seen in practice in section 3 (fig. 2). In other geometries, such as symmetry-broken fibres, it is possible that there exist narrow resonances where the transverse modes are changing significantly also in their nature, but such cases were not considered in this work. The resulting propagation equation maintains the same structure of Eq. (10) if the functions $\tilde{G}(z, \omega)$ are substituted with $\bar{G}_{m}(z, \omega)=\tilde{G}_{m}(z, \omega) \cdot \tilde{A}_{e f f, m}(\omega)^{-1 / 4}$, hence maintaining the same numerical complexity.

\section{Numerical results}

The accuracy of the proposed EAA is checked against a GQ method and compared to the CO approximation. The GQ approach directly evaluates the spatial integral in Eq. (5) on a reduced grid: its accuracy is set by the finesse of the grid with respect the mode profiles' smoothness. The GQ, described in detail for example in [14], is assumed to be an exact method if the grid is fine enough. The GQ results reported in this section are converged, in the sense that increasing the grid finesse has been checked to produce a negligible change in the output spectrum of the simulation. When a GQ simulation is converged, it is considered to be exact. The used grid parameters are $n_{r}=15$ radial steps, $n_{\theta}=7$ angular steps and $r_{\max }=3 a$ being the radial extent of the grid.

There can be two issues with the GQ approach. The first, most important reason is that the complexity is unfavourable in the few-modes regime; as will be seen for the two reported test cases, the GQ method takes significantly longer than the $\mathrm{CO}$, while the EAA is comparable to the latter. The second reason is that the GQ method is based on an assumption of smoothness of the mode profiles, which can be weaker for example in photonic crystal fibres, where the mode profiles conform to the lattice structure to some degree. This renders the GQ results approximate, as its results cannot be completely converged.

The use case for the EAA is then a few-mode fibre. In particular, the focus of this work on weakly-guiding fibres. Step-index and parabolic-index fibres with strong contrasts and practical core sizes are necessarily guiding a large number of modes; the unfavourable scaling of the overlap-based methods $\left(O\left(M^{4}\right)\right.$ where M is the number of modes) makes it so that the GQ is superior in efficiency, rendering the EAA uninteresting. Furthermore, the strong modal dispersion makes it more challenging to have non-trivial multimode nonlinear interactions. Microstructured fibres with strong contrasts and few-modes at optical frequencies can exist, and testing these geometries could be explored in future work.

Two different fibres have been tested, one step-index and one parabolic-index fibre; the former has $\Delta n=0.005$ while the latter has $\Delta n=0.01$. In both fibres the $L P_{1,1}$ mode group is cutoff close to the central wavelength of $\lambda_{0}=1550 \mathrm{~nm}$, and the dispersion parameter $D$ is not large $(|D|<25 \mathrm{ps} / \mathrm{nm}-\mathrm{km})$ for all modes at the centre wavelength.

The modes are calculated with a Finite Difference (FD) grid on a quarter of the total domain, and considering even/odd symmetries with respect to the centre. The simulations considered only the first 6 modes, ordered by the highest effective index. In the Linearly Polarized (LP) approximated modes, these would be the $L P_{0,1}$ and the $L P_{1,1}$ mode groups; the used FD modes are anyway full vector modes.

FD modes inherently carry an error due to the grid finesse $\delta x$ and an error due to the finite grid width $W$. The former of the two is seen to become more significant at lower wavelengths, where the modes are more confined, while the latter is crucial close to cutoffs, when the expanding 
modes feel the zero-field boundary condition of the grid. All the three methods (EAA, CO, GQ) are fed the same input fields, ensuring that these errors are not affecting the comparison of the simulation outputs. More in general, the three simulation methods are using the same parameters (modes, input wavelength and power, pulse shape, ...) so that the comparison is strictly on the simulation approach. Lastly, as can be seen in fig. 2's bottom panel, the cutoff is handled by forcing a linear fit from the last available frequency point before the cutoff to the predicted cutoff, forcing the overlap integrals to zero when an involved mode is cutoff. In other words, the data is fit with a cubic interpolation wherever possible, but then a linear fit smooths out the transition to the zero value of the overlap integral with a cutoff mode. Were the FD grid infinitely extending, this would not be necessary, as the overlap would smoothly die out as the mode leaks in the cladding. Proper handling of this is outside the scope of this work, and would again have no effect on the comparison of the methods' performance, as this is applied in both the EAA and the GQ.

\subsection{Parabolic Index Fibre}

The parabolic-index fibre in this section is chosen to have the $L P_{1,1}$ mode group cutoff at approximately $1.65 \mu \mathrm{m}$. The index distribution is of the kind

$$
n_{p}(r)= \begin{cases}n_{c}\left(1-\left(r / r_{0}\right)^{2}\right)^{0.5} & r \leq r_{c} \\ n_{c l} & r>r_{c}\end{cases}
$$

where the parameter $r_{0}$ is set by the continuity of the index at $r=r_{c}$. The chosen design uses $n_{c}=1.45, n_{c l}=1.44$ and $r_{c}=5.5 \mu \mathrm{m}$. The input pulse is a transform-limited gaussian pulse of FWHM $\tau_{0}=0.1 \mathrm{ps}$ and $\lambda_{0}=1.55 \mu \mathrm{m}$, with a peak power of $900 \mathrm{~kW}$ evenly distributed over the 6 modes, all in phase at the input. The simulation is run for $2 \mathrm{~cm}$, enough for a small DWG peak in the two polarization states of the fundamental mode to appear at around $701 \mathrm{~nm}$ for the $\mathrm{CO}$, $730 \mathrm{~nm}$ for the EAA and $731 \mathrm{~nm}$ for the GQ. It is found that the output of the CO is dramatically wrong, mainly due to its negligence of the modal cutoff at $1.65 \mu \mathrm{m}$. The EAA represents the spectrum shape with a remarkable degree of accuracy, compared to the GQ, and is around 20 times faster (discussed in detail later). The difference in the power spectra is $6 \%$ between the EAA and the GQ and $72 \%$ between the CO and the GQ.

Fig. 2 reports two representative examples of the overlap integrals in this fibre, calculated with the different methods and compared to the exact solution. The values are renormalized to the fundamental mode overlap $\Gamma_{0,0,0,0}$ (with all frequency arguments set to $\omega_{0}$ ); all the overlap integrals in the figure are calculated with the four frequency arguments being equal. In this notation, the mode 0 refers to one of the polarization states of the fundamental mode while the mode 1 refers to one of the polarization states of one of the orientations of the $L P_{1,1}$ mode. It is seen that the $\mathrm{CO}$ neglects a significant variation in the overlap integrals (the power distributed around $\lambda_{0}$ in fig. 1 extends approximately from $1.5 \mu \mathrm{m}$ to $2 \mu \mathrm{m}$ ), while the EAA and the GQ model this rather accurately. Fig. 3 reports for the overlap integral in the bottom of fig. 2 two examples where the frequency arguments are not all equal. In this case it is found that the EAA loses accuracy; the output spectrum seems to be anyway not strongly affected by this.

\subsection{Step Index Fibre}

The step-index fibre was chosen to have the $L P_{1,1}$ modal cutoffs at approximately $1.85 \mu m$. This is achieved with a core of $a=6 \mu \mathrm{m}, n_{c l}=1.44$ and $n_{c}=1.445$. The input pulse and all the other parameters are unchanged from the parabolic-index fibre. The output power spectrum is reported in fig. 4. As expected, the approximation performs better here than in the PIF. The overlap integrals involving modes of the same group are found to be better represented by the EAA; in particular the overlaps of the kind $\Gamma_{m, m, m, m}$ are exact. These terms become the main contributors to the spectrum evolution once the different mode groups drift away due to their different group 


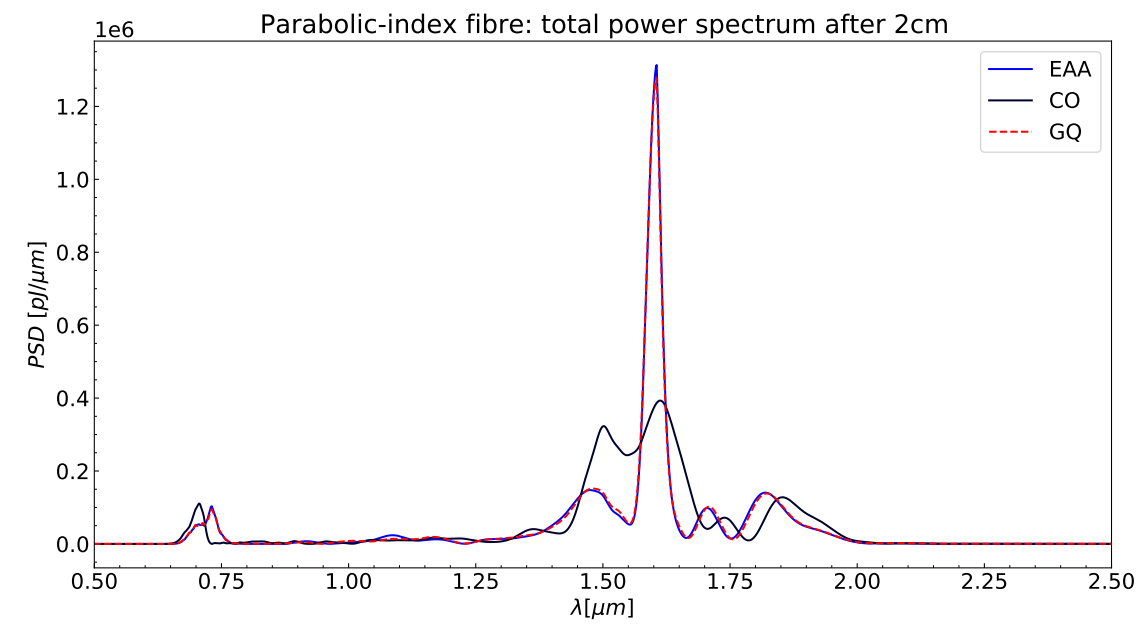

Fig. 1. Power spectral density, summed over the 6 modes, for the three methods in a parabolic-index fibre of $r_{c}=5.5 \mu \mathrm{m}$ and $\Delta n=0.01$. The total simulation wavelength grid $(0.4-4.0 \mu \mathrm{m})$ is not shown completely. Good agreement between EAA and GQ is found on the total spectrum and individual modes' spectra (not reported here). This spectrum showcases the increase in efficiency of the EAA over the GQ with an acceptable loss of accuracy.
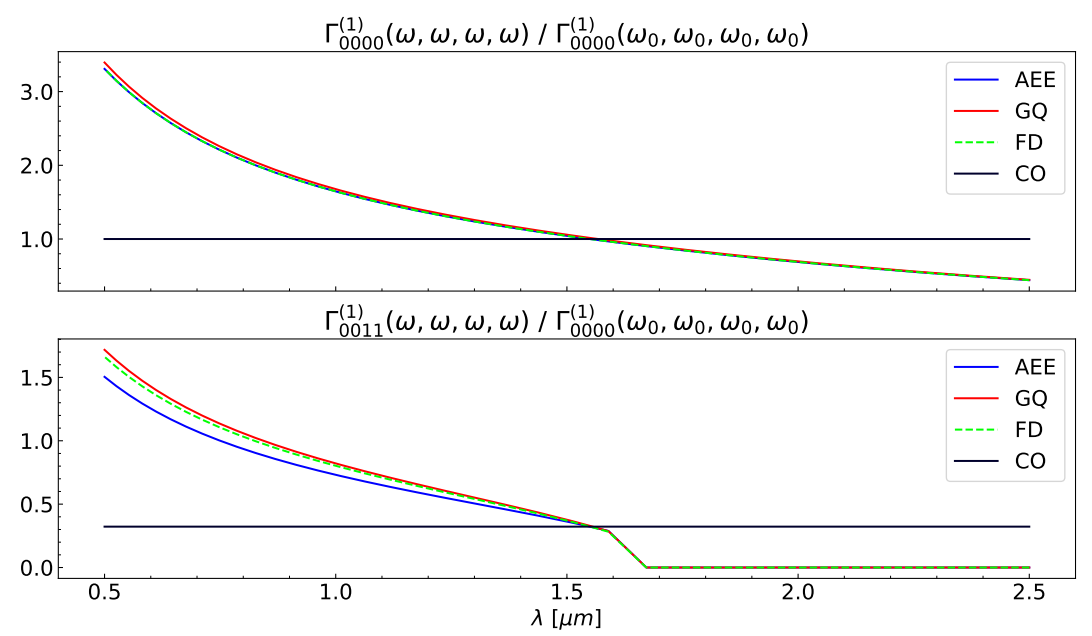

Fig. 2. Representative examples of the overlap integral $\Gamma^{(1)}\left(\omega=\omega_{1}=\omega_{2}=\omega_{1}+\omega_{2}-\omega\right)$ for the PIF. The 'FD' curve is the exact overlap calculated integrating the FD modes at every frequency point.

velocities. It is known that a PIF has a lower degree of modal dispersion compared to a step-index geometry. The lower error figure is then justified by the shorter interaction distance of the $L P_{0,1}$ and $L P_{1,1}$ mode groups. In this case, the DWG peak is at $771 \mathrm{~nm}$ for the two dispersive methods and $770 \mathrm{~nm}$ for the $\mathrm{CO}$, and it is again generated only in the fundamental mode. The difference in the power spectra is $1 \%$ between the EAA and the GQ and 55\% between the CO and the GQ. 

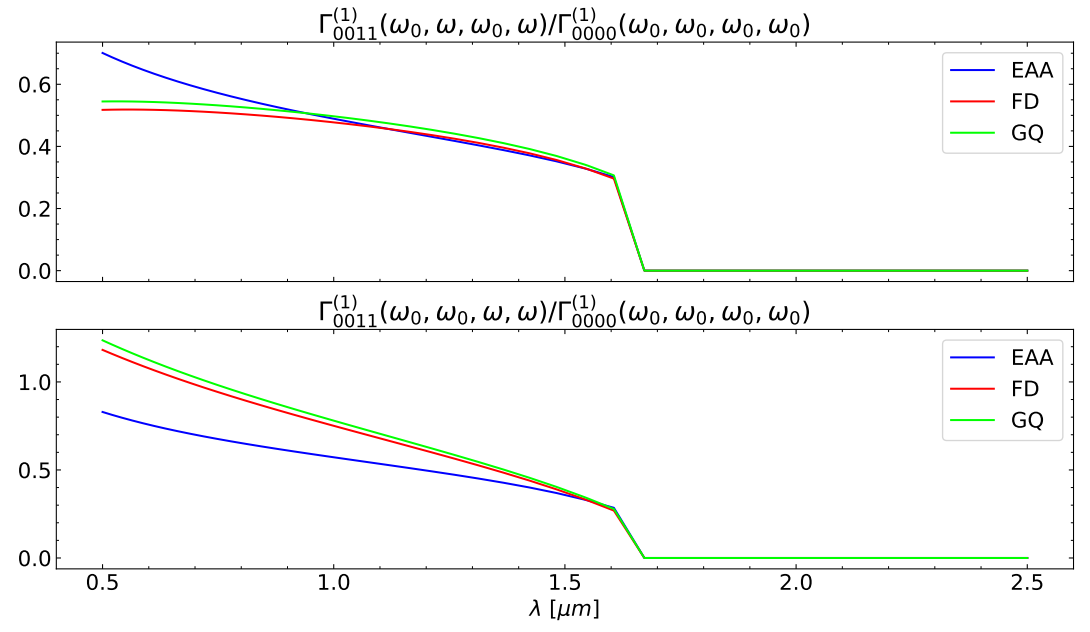

Fig. 3. Representative examples of the overlap integral $\Gamma_{0011}^{(1)}$ when the frequency arguments are not all equal. Recall that the fourth frequency argument is fixed to $\omega_{1}+\omega_{2}-\omega$.

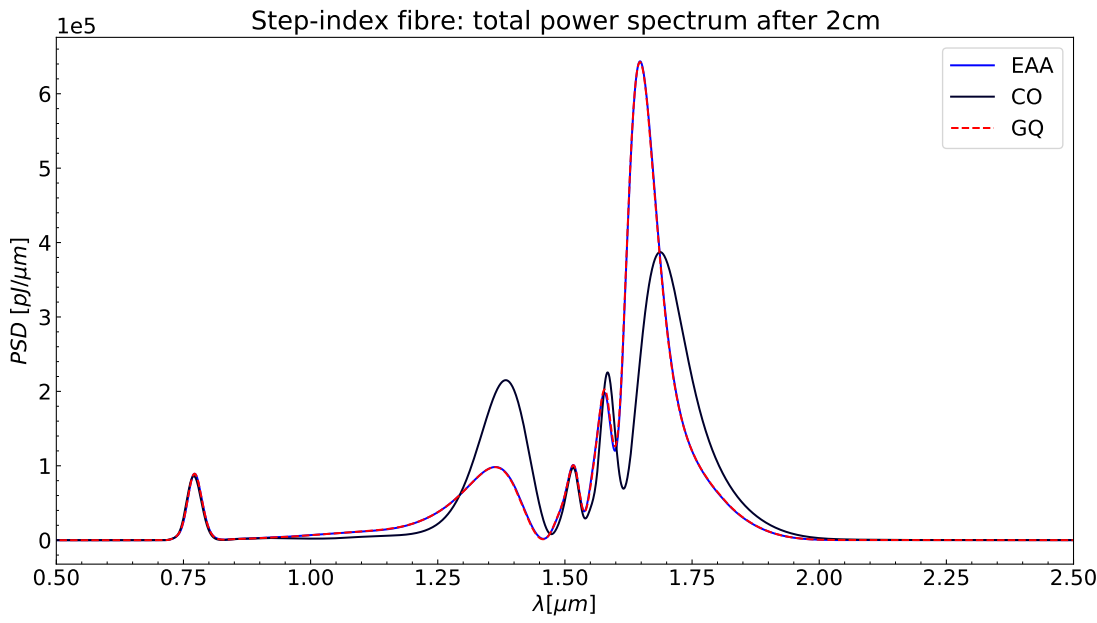

Fig. 4. Power spectral density, summed over the 6 modes, for the three methods in a step-index fibre of $a=6 \mu m$ and $\Delta n=0.005$. The total simulation wavelength grid $(0.4-4.0 \mu \mathrm{m})$ is not shown completely. Good agreement between EAA and GQ is also found on the individual modes' spectra (not reported here).

\subsection{Comparison of the execution time}

Due to the large effective areas close to the modal cutoff at $1.65 \mu \mathrm{m}$ and $1.85 \mu \mathrm{m}$, the GQ grid needs to be fairly large (in terms of $r_{\max }$ ) to have converged results. In fig. 1 and fig. 4, the GQ grid parameters were $n_{r}=15, n_{\theta}=7$ and $r_{\max }=3 a$. This is a slightly conservative choice, justified by the requirement to assess the accuracy of the EAA without a leftover convergence error for the GQ. 
Running a GQ simulation required up to 2 hours, hence making it impractical to average the execution time over many runs. Furthermore, the use of a nonlinear integrator (dopri5 in scipy.integrate.ode) with variable stepsize influences the run time comparison between simulations whose results are different, for example slowing simulations down when the peak power is higher, due to the need for a finer stepping to reach the target level of accuracy. Considering the PIF spectrum, this means that the EAA and GQ are slowed down with respect to the CO because the peak powers are higher in the former two. With this in mind, the time comparison is performed on a single call to the subroutine evaluating the nonlinear operator, i.e. the left hand side of Eq. (5). The more thorough complexity comparison in [14] for the GQ and CO remains valid for the EAA, as its complexity is the same of the CO.

The used implementation of the CO and EAA is iterating through the non-null overlap integrals, rather than the direct implementation of four nested cycles on the modal indices, which in these test cases more or less cuts the execution time in half. EAA and CO differ only in the preparation of the $\bar{G}$ from the $\tilde{G}$ functions in the initialization of the subroutine. As a result of this, the call time of the nonlinear operator function in the both cases is increased by only $\leq 7 \%$ averaged over 10000 calls. This value is dependent on the exact simulation parameters, as for example the ratio of non-null overlap integrals, but it is expected to always be a modest penalty. On the other hand, the GQ call time is at least 13 times longer than the $\mathrm{CO}$ (for both fibres). This value is found to be strongly hardware dependent: for the same simulation, with the same Python version (3.8), an i7 CPU produced a 13.5 ratio while an i5 produced a 26.5 . In any case, the GQ is significantly slower.

Part of the increased efficiency of the CO and EAA with respect to the GQ method is due to the ability to know which overlap integrals are zero before calculating the relative term in the triple sum in Eq. 10. Implementing this knowledge within the GQ method is impossible, because the overlap integrals are never explicitly evaluated. As mentioned earlier, this can roughly cut the execution time in half.

The run times for one run of the two simulations reported earlier are also reported in fig. 5; these are not averaged, but during this work they have been seen to be quite consistent with each other. The equivalent data is not reported for the PIF, but the output time values are $289 s$, $330 s$ and $6093 s$ for CO, EAA and GQ respectively. The total execution times are affected by the mentioned variable step-size, but also from auxiliary functions in the simulation, as for example saving debug information, so that the total execution time ratios are differing from the nonlinear operator function ratios.

\section{Discussion}

The Effective Area Approximation proposed in this work is a significant accuracy improvement over the $\mathrm{CO}$ method in situations where the power is distributed over a few modes and a large spectrum, especially if modal cutoffs are present, while retaining its lower complexity (compared to the GQ). This is also found in the reported overlap integrals. The EAA is modelling the dispersion of the integrals accurately, albeit progressively losing accuracy moving away from $\lambda_{0}$. The complexity penalty for including mode profile dispersion through the EAA is practically negligible.

A straightforward improvement would be to distinguish $A_{\text {eff }}$ and $A_{\text {eff }}^{\prime}$ for the two different conjugations of the overlap integrals $\Gamma^{(1)}$ and $\Gamma^{(2)}$. This was seen to be not worth the complexity penalty in weakly-guiding fibres, but might become more important in geometries with stronger index contrasts.

The test cases, a step-index fibre and a parabolic-index fibre, consider modes with simple and smooth profiles. The overlap integrals then have a simple frequency dependence (they decrease monotonically for longer wavelengths until cutoff), which is captured accurately by the effective 


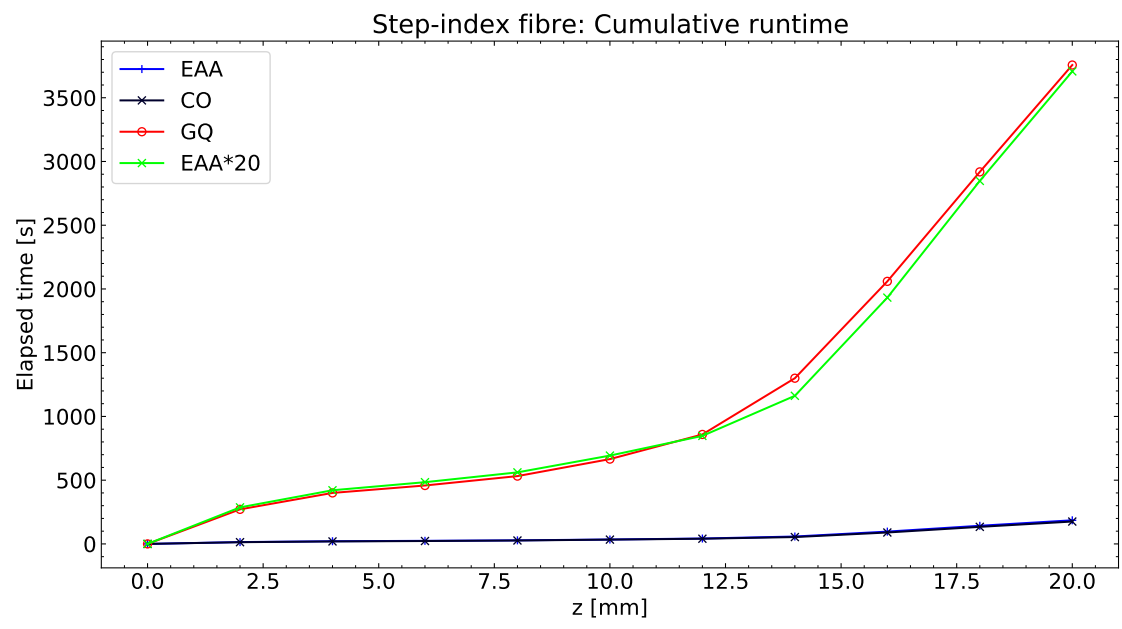

Fig. 5. Elapsed time for the SIF simulation, whose output is reported in fig. 4. The values at $z=20 \mathrm{~mm}$ are $176 s$ for the CO, $188 s$ for the EAA and $3758 s$ for the GQ. The green line reports the EAA line, rescaled by a factor 20, to show that the curve shape is quite similar to the GQ.

areas of the modes. Other geometries, as for example a photonic crystal fibre, are problematic for the GQ scheme, as they require large grids and the positioning of the grid points is not trivial. In these cases, should the overlap integrals behave similarly to the tested simpler geometries, the EAA could be more convenient than the GQ scheme. This has not been tested currently but could be explored in future work.

\section{Conclusions}

To conclude, an approximation of the overlap integrals' frequency dependence based on their effective area is proposed. The approximation conserves the photon number. The approximation is tested against a simpler approach using constant overlap integrals and an exact gaussian quadrature approach, for two weakly guiding fibres. The results show that this approximation performs significantly better than the constant overlaps, with little computational overhead and excellent agreement with the heavier approach. Further investigation on validity of the approximation is required for different fibre geometries.

\section{Disclosures}

The authors declare no conflicts of interest.

\section{Data Availability}

Data underlying the results presented in this paper are not publicly available at this time but may be obtained from the authors upon reasonable request. 


\section{Appendix: Photon number conservation}

It is found that the EAA conserves the photon number during propagation. For our purposes it is sufficient to define the photon number as

$$
N_{P}=\int d \omega \frac{|G(\omega)|^{2}}{\omega}
$$

In traditional single-mode NLSE formulations with constant $A_{\text {eff }}, N_{P}$ may be conserved with proper inclusion of the self-steepening term, when using the field normalization adopted in this work, Eq. 3. Note that different field normalizations can lead to different conservation laws, e.g. the normalizations used in $[17,21]$ leads to conservation of the quantity

$$
N_{P}^{\prime}=\int d \omega n_{e f f}(\omega) \frac{|G(\omega)|^{2}}{\omega}
$$

Inclusion of a frequency-dependent $A_{e f f}$ leads to a further correction if it is done as in [21], but not if it is done as in e.g. [17].

The photon number conservation can also be used to verify that a simulation is well-behaved by checking that it is numerically respected. The two simulations reported in this work have a normalized variation of the photon number from input to output of $2 \cdot 10^{-6}$ for the PIF and $<1 \cdot 10^{-6}$ for the SIF. These values are comparable to the ones for the CO and GQ.

To begin with, the z-derivative of the photon number is, neglecting normalization factors, defined as

$$
\frac{\partial N_{P}}{\partial z}=\frac{\partial}{\partial z} \sum_{m} \int d \omega \frac{|G(\omega)|^{2}}{\omega}=\int d \omega \sum_{m} \frac{G_{m}^{*} \partial_{z} G_{m}+G_{m} \partial_{z} G_{m}^{*}}{\omega}=2 R e \int d \omega \sum_{m} \frac{G_{m}^{*} \partial_{z} G_{m}}{\omega}
$$

The objective of this section is to prove that this derivative is zero. The partial derivatives are given by the NLSE

$$
\begin{aligned}
\frac{\partial G_{m}(\omega)}{\partial z}= & -i \omega \epsilon_{0} \chi^{(3)} \exp \left(i \beta_{m}(\omega) z\right) \sum_{n, p, q} \frac{1}{(2 \pi)^{2}} \cdot\left(2 \frac{f_{m n p q}^{(1)}}{A_{\text {eff,m }}^{1 / 4}(\omega)} \cdot\right. \\
& \int d \omega_{1} d \omega_{2} \frac{\tilde{G}_{n}\left(\omega_{1}\right)}{A_{\text {eff,n }}^{1 / 4}\left(\omega_{1}\right)} \frac{\tilde{G}_{p}\left(\omega_{2}\right)}{A_{\text {eff,p }}^{1 / 4}\left(\omega_{2}\right)} \frac{\tilde{G}_{q}^{*}\left(\omega_{1}+\omega_{2}-\omega\right)}{A_{\text {eff }, q}^{1 / 4}\left(\omega_{1}+\omega_{2}-\omega\right)} \cdot R\left(\omega-\omega_{1}\right)+ \\
& \left.\left(1-f_{R}\right) \frac{f_{m n p q}^{(2)}}{A_{\text {eff }, m}^{1 / 4}(\omega)} \cdot \int d \omega_{1} d \omega_{2} \frac{\tilde{G}_{n}^{*}\left(\omega_{1}\right)}{A_{e f f, n}^{1 / 4}\left(\omega_{1}\right)} \cdot \frac{\tilde{G}_{p}\left(\omega_{2}\right)}{A_{\text {eff }, p}^{1 / 4}\left(\omega_{2}\right)} \cdot \frac{\tilde{G}_{q}\left(\omega_{1}+\omega_{2}-\omega\right)}{A_{e f f, q}^{1 / 4}\left(\omega_{1}+\omega_{2}-\omega\right)}\right)
\end{aligned}
$$

Consider now only the second, Kerr-only term. Its contribution to Eq. (16) can be written as

$$
\begin{aligned}
\frac{\partial N_{P}}{\partial z} \sim \operatorname{Re}\left[-i \iiint d \omega d \omega_{1} d \omega_{2} \sum_{m, n, p, q} f_{m, n, p, q}^{(2)} \cdot\right. \\
\left.\quad \frac{G_{m}^{*}(\omega) e^{i \beta_{m}(\omega) z}}{A_{\text {eff }, m}^{1 / 4}(\omega)} \frac{G_{n}^{*}\left(\omega_{1}\right) e^{i \beta_{n}\left(\omega_{1}\right) z}}{A_{\text {eff }, n}^{1 / 4}\left(\omega_{1}\right)} \frac{G_{p}\left(\omega_{2}\right) e^{-i \beta_{p}\left(\omega_{2}\right) z}}{A_{\text {eff,p }}^{1 / 4}\left(\omega_{2}\right)} \frac{G_{q}\left(\omega_{1}+\omega_{2}-\omega\right) e^{-i \beta_{q}\left(\omega_{1}+\omega_{2}-\omega\right) z}}{A_{\text {eff }, q}^{1 / 4}\left(\omega_{1}+\omega_{2}-\omega\right)}\right]
\end{aligned}
$$


First, it is found that the $m$ factor becomes the $\bar{G}$ function, analogously to the other three terms. Secondly, the symmetry in the approximation factor $f_{m, n, p, q}^{(2)}$ is seen from its definition:

$$
f_{m, n, p, q}^{(2)}=\left.\frac{\int d \mathbf{r}_{\perp} e_{m}^{*}(\omega) \cdot e_{n}^{*}\left(\omega_{1}\right) e_{p}\left(\omega_{2}\right) \cdot e_{q}\left(\omega_{1}+\omega_{2}-\omega\right)}{\left(A_{e f f, m}(\omega) A_{e f f, n}\left(\omega_{1}\right) A_{e f f, p}\left(\omega_{2}\right) A_{e f f, q}\left(\omega_{1}+\omega_{2}-\omega\right)\right)^{1 / 4}}\right|_{\omega=\omega_{1}=\omega_{2}=\omega_{0}}
$$

The modal fields can be assumed to be of the kind $\mathbf{e}=\left(e_{x}, e_{y}, i \cdot e_{z}\right)$ where the single components are real, without loss of generality. Under this assumption

$$
\mathbf{e}_{m} \cdot \mathbf{e}_{n}=\mathbf{e}_{m}^{*} \cdot \mathbf{e}_{n}^{*} \quad \mathbf{e}_{m}^{*} \cdot \mathbf{e}_{n}=\mathbf{e}_{m} \cdot \mathbf{e}_{n}^{*}
$$

which implies $f_{m, n, p, q}^{(2)}=f_{p, q, m, n}^{(2)}$. Consider then the component of the sum in the photon number $c_{m, n, p, q}$

$$
\begin{array}{r}
c_{m, n, p, q}=\iiint_{m, n, p} d \omega d \omega_{1} d \omega_{2} \bar{G}_{m}^{*}(\omega) \bar{G}_{n}^{*}\left(\omega_{1}\right) \bar{G}_{p}\left(\omega_{2}\right) \bar{G}_{q}\left(\omega_{1}+\omega_{2}-\omega\right) \\
c_{m}^{*} \iiint_{\int} d \omega d \omega_{1} d \omega_{2} \bar{G}_{m}(\omega) \bar{G}_{n}\left(\omega_{1}\right) \bar{G}_{p}^{*}\left(\omega_{2}\right) \bar{G}_{q}^{*}\left(\omega_{1}+\omega_{2}-\omega\right)= \\
\iiint d \omega d \omega_{1} d \omega_{2} \bar{G}_{m}(\omega) \bar{G}_{n}\left(\omega_{1}\right) \bar{G}_{p}^{*}\left(\omega^{\prime}\right) \bar{G}_{q}^{*}\left(\omega_{1}+\omega^{\prime}-\omega\right)= \\
\iiint d \omega d \omega_{1} d \omega_{2} \bar{G}_{m}\left(\omega_{2}\right) \bar{G}_{n}\left(\omega_{1}\right) \bar{G}_{p}^{*}\left(\omega^{\prime}\right) \bar{G}_{q}^{*}\left(\omega_{1}+\omega^{\prime}-\omega_{2}\right)= \\
\iiint d \omega d \omega_{1} d \omega_{2} \bar{G}_{m}\left(\omega_{2}\right) \bar{G}_{n}\left(\omega_{1}^{\prime}+\omega_{2}-\omega^{\prime}\right) \bar{G}_{p}^{*}\left(\omega^{\prime}\right) \bar{G}_{q}^{*}\left(\omega_{1}^{\prime}\right)=c_{p, q, m, n}
\end{array}
$$

where, in order, the following variable substitutions are performed: $\omega^{\prime}=\omega_{2}, \omega_{2}=\omega, \omega_{1}^{\prime}=$ $\omega_{1}+\omega^{\prime}-\omega_{2}$ and eventually the primes are dropped. Looking now at the sum in Eq. (18), these two factors will sum as

$$
\operatorname{Re}\left(-i\left(f_{m, n, p, q}^{(2)} \cdot c_{m, n, p, q}+f_{p, q, m, n}^{(2)} \cdot c_{p, q, m, n}\right)\right)=\operatorname{Re}\left(-2 i \operatorname{Re}\left(f_{m, n, p, q}^{(2)} \cdot c_{m, n, p, q}\right)\right)
$$

giving no contribution. The inner real part is not to be mistaken with the outer one, which comes from the sum with the complex conjugate of $\partial_{z} G_{m} G_{m}^{*}$ in the first integral in Eq. (16). Up to now it was neglected that terms like for example $c_{0,0,0,0}$ will not be summed to their complex conjugate in the four mode index summations; these should be handled separately. The issue lies in general in all the terms $c_{m, n, m, n}$. Trivially, both the $c_{m, n, m, n}$ and the $f_{m, n, m, n}$ factors are special cases of the $c_{m, n, p, q}$ and $f_{m, n, p, q}$ symmetries. Since it can be shown that the term's complex conjugate coincides with itself, then it is real valued and it will not contribute either. This means then that all the Kerr-only contributions cancel out and they conserve the photon number.

For the Raman term instead

$$
\begin{aligned}
\frac{\partial N_{P}}{\partial z} & \sim \operatorname{Re}\left[-i \iiint d \omega d \omega_{1} d \omega_{2} \sum_{m, n, p, q} f_{m, n, p, q}^{(1)} R\left(\omega-\omega_{1}\right) \cdot\right. \\
& \left.\frac{G_{m}^{*}(\omega) e^{i \beta_{m}(\omega) z}}{A_{e f f, m}^{1 / 4}(\omega)} \frac{G_{n}\left(\omega_{1}\right) e^{-i \beta_{n}\left(\omega_{1}\right) z}}{A_{e f f, n}^{1 / 4}\left(\omega_{1}\right)} \frac{G_{p}\left(\omega_{2}\right) e^{-i \beta_{p}\left(\omega_{2}\right) z}}{A_{e f f, p}^{1 / 4}\left(\omega_{2}\right)} \frac{G_{q}^{*}\left(\omega_{1}+\omega_{2}-\omega\right) e^{i \beta_{q}\left(\omega_{1}+\omega_{2}-\omega\right) z}}{A_{e f f, q}^{1 / 4}\left(\omega_{1}+\omega_{2}-\omega\right)}\right]
\end{aligned}
$$


The symmetry which is relevant now is that $f_{m, n, p, q}^{(1)}=f_{n, m, q, p}^{(1)}$, while on the other hand the frequency triple integral will again produce a conjugate sign:

$$
\begin{gathered}
c_{m, n, p, q}=\iiint d \omega d \omega_{1} d \omega_{2} \bar{G}_{m}^{*}(\omega) \bar{G}_{n}\left(\omega_{1}\right) \bar{G}_{p}\left(\omega_{2}\right) \bar{G}_{q}^{*}\left(\omega_{1}+\omega_{2}-\omega\right) R\left(\omega-\omega_{1}\right) \\
c_{m, n, p, q}^{*}=\iiint d \omega d \omega_{1} d \omega_{2} \bar{G}_{m}(\omega) \bar{G}_{n}^{*}\left(\omega_{1}\right) \bar{G}_{p}^{*}\left(\omega_{2}\right) \bar{G}_{q}\left(\omega_{1}+\omega_{2}-\omega\right) R^{*}\left(\omega-\omega_{1}\right)= \\
\iiint d \omega d \omega_{1} d \omega_{2} \bar{G}_{n}^{*}(\omega) \bar{G}_{m}\left(\omega_{1}\right) \bar{G}_{q}\left(\omega_{2}\right) \bar{G}_{p}^{*}\left(\omega_{1}+\omega_{2}-\omega\right) R^{*}\left(\omega_{1}-\omega\right)=c_{n, m, q, p}
\end{gathered}
$$

where the last equality is valid if and only if $R(\omega)=R^{*}(-\omega)$. From the properties of the Fourier transform, it follows that the condition is true when the time response is real valued, which is assumed to be the case as the electric field is real valued in time. Therefore, similarly to the Kerr only term, when summing over all the mode combinations, the contributions cancel out. The special combinations are those that do not have a complex conjugate in the index switch i.e. the terms $m, m, p, p$. It follows quite easily, with a similar argument to the Kerr term, that these are real valued in the first place.

To sum up, all the terms have been shown to be either real valued or cancelling with a companion complex conjugate term. Going back to Eq. (16), this implies that the photon number is conserved.

\section{References}

1. C. Antonelli, M. Shtaif, and A. Mecozzi, "Modeling of nonlinear propagation in space-division multiplexed fiber-optic transmission," J. Light. Technol. 34, 36-54 (2016).

2. J. M. Dudley, G. Genty, and S. Coen, "Supercontinuum generation in photonic crystal fiber," Rev. Mod. Phys. 78, 1135-1184 (2006).

3. N. Akhmediev and M. Karlsson, "Cherenkov radiation emitted by solitons in optical fibers," Phys. Rev. A 51, 2602-2607 (1995).

4. G. Prabhakar, P. Gregg, L. Rishoj, P. Kristensen, and S. Ramachandran, "Octave-wide supercontinuum generation of light-carrying orbital angular momentum," Opt. Express 27, 11547-11556 (2019).

5. J. Lægsgaard, "Power scaling of dispersive-wave generation in higher-order optical fiber modes," J. Opt. Soc. Am. B 37, 2637-2645 (2020).

6. J. Demas, P. Steinvurzel, B. Tai, L. Rishøj, Y. Chen, and S. Ramachandran, "Intermodal nonlinear mixing with Bessel beams in optical fiber," Optica 2, 14-17 (2015).

7. S. R. Petersen, T. T. Alkeskjold, C. B. Olausson, and J. Lægsgaard, "Intermodal and cross-polarization four-wave mixing in large-core hybrid photonic crystal fibers," Opt. Express 23, 5954-5971 (2015).

8. L. G. Wright, S. Wabnitz, D. N. Christodoulides, and F. W. Wise, "Ultrabroadband dispersive radiation by spatiotemporal oscillation of multimode waves," Phys. Rev. Lett. 115, 223902 (2015).

9. P. Aschieri, J. Garnier, C. Michel, V. Doya, and A. Picozzi, "Condensation and thermalization of classsical optical waves in a waveguide," Phys. Rev. A 83, 033838 (2011).

10. L. G. Wright, Z. Liu, D. A. Nolan, M.-J. Li, D. N. Christodoulides, and F. W. Wise, "Self-organized instability in graded-index multimode fibres," Nat. Photonics 10, 771+ (2016).

11. K. Krupa, A. Tonello, B. M. Shalaby, M. Fabert, A. Barthelemy, G. Millot, S. Wabnitz, and V. Couderc, "Spatial beam self-cleaning in multimode fibres," Nat. Photonics 11, 237-241 (2017).

12. C. Monterola and C. Saloma, "Solving the nonlinear schrodinger equation with an unsupervised neural network," Opt. Express 9, 72-84 (2001).

13. F. Poletti and P. Horak, "Description of ultrashort pulse propagation in multimode optical fibers," J. Opt. Soc. Am. B 25, 1645-1654 (2008).

14. J. Lægsgaard, "Efficient simulation of multimodal nonlinear propagation in step-index fibers," J. Opt. Soc. Am. B 34, 2266-2273 (2017).

15. J. Lægsgaard, "Full-vectorial multimode nonlinear simulations on a real-space fourier-gauss grid,” J. Opt. Soc. Am. B 36, 2235-2243 (2019).

16. L. Rishøj, B. Tai, P. Kristensen, and S. Ramachandran, "Soliton self-mode conversion: revisiting raman scattering of ultrashort pulses," Optica 6, 304-308 (2019).

17. J. Lægsgaard, "Mode profile dispersion in the generalized nonlinear schrödinger equation,” Opt. Express 15, 16110-16123 (2007).

18. M. Kolesik, E. Wright, and J. Moloney, "Simulation of femtosecond pulse propagation in sub-micron diameter tapered fibers," Appl. Phys. B 79, 293-300 (2004). 
19. D. Hollenbeck and C. D. Cantrell, "Multiple-vibrational-mode model for fiber-optic raman gain spectrum and response function," J. Opt. Soc. Am. B 19, 2886-2892 (2002).

20. S. A. Vahid and T. M. Monro, "A full vectorial model for pulse propagation in emerging waveguides with subwavelength structures part i: Kerr nonlinearity," Opt. Express 17, 2298-2318 (2009).

21. K. J. Blow and D. Wood, "Theoretical description of transient stimulated raman scattering in optical fibers," IEEE J. Quantum Electron. 25, 2665-2673 (1989). 